\title{
PAUF promotes adhesiveness of pancreatic cancer cells by modulating focal adhesion kinase
}

\author{
Yangsoon Lee ${ }^{1}$, Su Jin Kim ${ }^{1,2}$, Hye Jin Min ${ }^{1}$, \\ Ji Yoon $\mathrm{Jo}^{1,2}$, Eun Hye Park \\ and Sang Seok Koh ${ }^{1,2,3}$ \\ ${ }^{1}$ Therapeutic Antibody Research Center \\ Korea Research Institute of Bioscience and Biotechnology \\ Daejeon 305-806, Korea \\ ${ }^{2}$ Department of Functional Genomics \\ University of Science \& Technology \\ Daejeon 305-333, Korea \\ ${ }^{3}$ Corresponding author: Tel, 82-42-860-4125; \\ Fax, 82-42-860-4149; E-mail, sskoh@kribb.re.kr \\ DOI 10.3858/emm.2011.43.5.030
}

Accepted 28 March 2011

Available Online 5 April 2011

Abbreviations: ECM, extracellular matrix; FAK, focal adhesion kinase; PAUF, pancreatic adenocarcinoma up-regulated factor

\begin{abstract}
Pancreatic cancer is a notorious disease with a poor prognosis and low survival rates, which is due to limited advances in understanding of the molecular mechanism and inadequate development of effective treatment options over the decades. In previous studies, we demonstrated that a novel soluble protein named pancreatic adenocarcinoma up-regulated factor (PAUF) acts on tumor and immune cells and plays an important role in metastasis and progression of pancreatic cancer. Here we show that PAUF promotes adhesiveness of pancreatic cancer cells to various extracellular matrix (ECM). Our results further support a positive correlation of activation and expression of focal adhesion kinase (FAK), a key player in tumor cell metastasis and survival, with PAUF expression. PAUF-mediated adhesiveness was significantly attenuated upon blockade of the FAK pathway. Moreover, PAUF appeared to enhance resistance of pancreatic cancer cells to anoikis via modulation of FAK. Our results suggest that PAUF-mediated FAK activation plays an important role in pancreatic cancer progression.
\end{abstract}

Keywords: adhesion; anoikis; FAK; PAUF; pancreatic cancer; signaling

\section{Introduction}

Pancreatic cancer, the fourth leading cause of cancer-induced mortality in both men and women, has an exceedingly poor prognosis, and is a major health issue in the developed world (Maitra and Hruban, 2008). An estimated 200,000 people develop and die from this disease each year (Ghaneh et al., 2007; Maitra and Hruban, 2008). The poor prognosis of pancreatic cancer is attributed to late presentation, aggressive local invasion, early metastasis, and poor response to chemotherapy (Duffy et al., 2003). Furthermore, retrospective studies have revealed a global increase in the overall mortality rate from pancreatic cancer, reflecting increasing incidence of pancreatic tumors (Garcea et al., 2005). The escalating incidence and poor prognosis of pancreatic cancer emphasize the need for methods to treat and methods to identify agents that can effectively target this disease.

Previously, we identified a novel secretory protein, pancreatic adenocarcinoma up-regulated factor (PAUF), which potentiates metastasis of pancreatic cancer cells via upregulation of migration and invasion (Kim et al., 2009; Lee et al., 2010; Cho et al., 2011). Analysis of the X-ray crystal structure revealed that PAUF is a mammalian lectin containing a $\beta$-prism motif normally found in plant lectins (Park et al., 2011). In cancer cells, PAUF acts in an autocrine manner to activate the ERK, JNK and AKT intracellular signaling cascades, resulting in increased expression of the genes involved in tumor progression and metastasis (Lee et al., 2010). Expression of CXCR4, a PAUF-inducing gene, is also positively correlated with PAUF expression in pancreatic cancer tissues from patients (Lee et al., 2010). In immune cells, PAUF interacts with the CXCR4-TLR2 complex and inhibits CXCR4-dependent TLR2-mediated NF$\kappa B$ activation in a paracrine manner (Park et al., 2011). These results suggest that PAUF enhances tumorigenic potential through facilitating tumor metastasis and escaping immuno-surveillance.

Metastasis of cancer cells is regarded as a complex, multistage process involving epithelialmesenchymal transition, invasion into and dissemination through the bloodstream or lymphatic system, and colonization at specific organs (Christofori, 2006; Steeg, 2006; Sahai, 2007). However, 
little is known about the molecular mechanisms controlling pancreatic cancer metastasis at present. Focal adhesion kinase (FAK), a non-receptor tyrosine kinase, is associated with diverse cellular functions, including adhesion, polarization, migration and survival (Frame et al., 2010). In cancer cells, activated FAK contributes to tumor progression and metastasis by enhancing motility, adhesiveness and resistance against apoptosis. In addition, overexpression of FAK is positively associated with tumor progression and poor prognosis (Owens et al., 1995; Lark et al., 2003; Sawai et al., 2005). FAK regulates the adhesive behavior of pancreatic cancer through activating the Ras-ERK signaling pathways (Sawai et al., 2005). Earlier, we showed that PAUF potentiates tumorigenicity via activation of multiple signaling pathways, including ERK, AKT and JNK (Lee et al., 2010).

Here we extend this previous work to show that PAUF has tumorigenic potential through regulation of the activity and expression of FAK. We propose that PAUF promotes the adhesiveness and survival of pancreatic cancer cells via modulation of the kinase.

\section{Results}

\section{PAUF promotes adhesion of pancreatic cancer cells}

Previously, we reported that PAUF enhances the migration and invasion of pancreatic cancer cells (Lee et al., 2010). In this study, we investigated whether PAUF influences the adhesiveness of pancreatic cancer cells to extracellular matrix (ECM). Compared with the respective controls, the adhesive activities of PAUF-overexpressing cell lines (Panc-1_PAUF and MiaPaca-2_PAUF) were significantly enhanced (Figure 1A), whereas those of PAUF-downregulated cell lines (CFPAC-1_ shPAUF and BxPC-3_shPAUF) were dramatically diminished (Figure 1B). The ECM molecules, fibronectin, collagen I and vitronectin, used for the adhesion assay, showed similar adhesion tendencies. The secretory nature of PAUF suggests that the protein affects the adhesiveness in an autocrine manner. As shown in Figure 1C, adhesiveness enhanced by overexpression of PAUF was attenuated upon treatment with an anti-PAUF antibody. The effect of anti-PAUF on adhesiveness was relatively low in PAUF-knockdown pancreatic cancer cells than its control cells (Figure 1D). These results support the theory that PAUF promotes adhesiveness of pancreatic cancer cells to $\mathrm{ECM}$ in an autocrine manner.
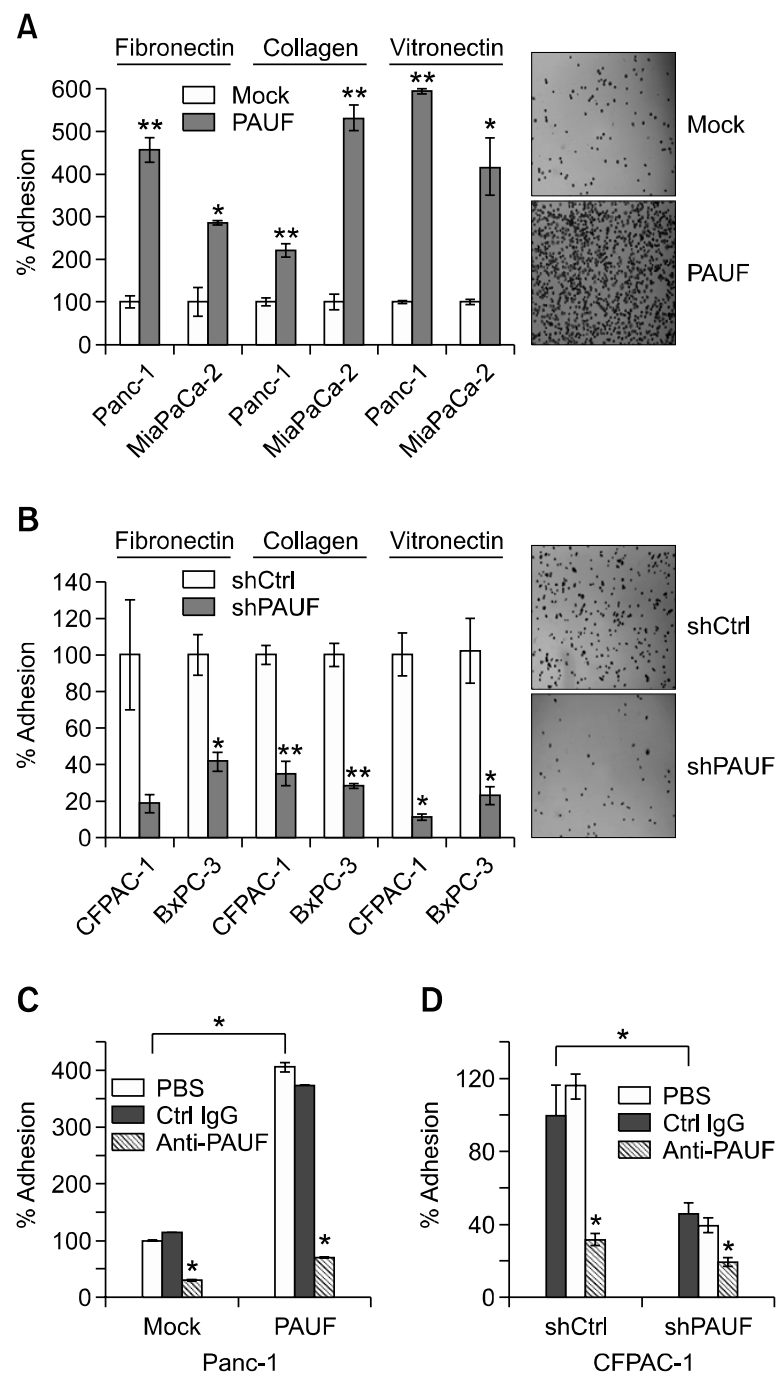

Figure 1. PAUF enhances the adhesiveness of pancreatic cells in vitro. $(A, B)$ The effects of PAUF overexpression (A) and knockdown (B) on cell adhesion were determined with the adhesion assay using fibronectin, collagen or vitronectin-coated plates. Cells were seeded in 96-well plates coated with each ECM molecule. After 20 min incubation at $37^{\circ} \mathrm{C}$, non-adherent cells were washed with PBS and then adherent cells were counted. (C, D) Adhesion activities of PAUF-overexpression (C) or knockdown (D) cells were measured in the presence of anti-PAUF (30 $\mu \mathrm{g} / \mathrm{ml}$ ), control antibody (heat-inactivated anti-PAUF, $30 \mu \mathrm{l} / \mathrm{ml}$ ), or PBS. Data represent mean values \pm SE. ${ }^{*}, P<0.05,{ }^{* *}, P<0.01$.

\section{PAUF facilitates FAK-Src signaling}

We examined the activation and expression of focal adhesion kinase (FAK) in cancer cells with modulated PAUF expression. Phosphorylation of FAK was increased in Panc-1_PAUF and MiaPaca2_PAUF cell lines, compared with controls, but diminished in CFPAC-1_shPAUF and BxPC-3 shPAUF cell lines (Figure 2A). Furthermore, stable overexpression of PAUF resulted in elevation of FAK expression. To regulate adhesion and migra- 
A
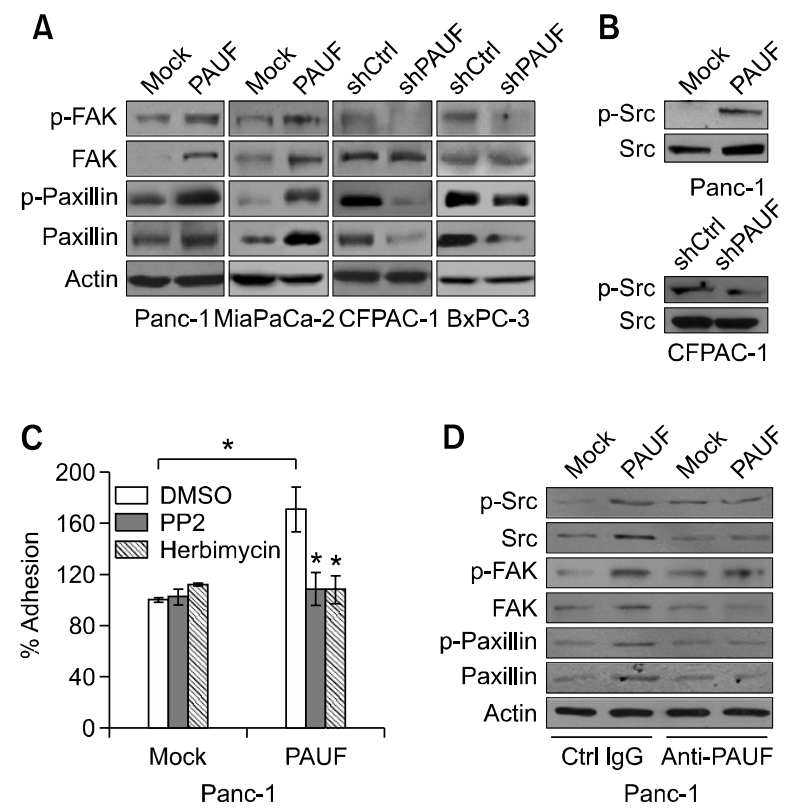

Figure 2. PAUF facilitates FAK-Src signalings. (A) Lysates were prepared from Panc-1_Mock (Mock), Panc-1_PAUF (PAUF), MiaPaCa-2 Mock (Mock), MiaPaCa-2 PAUF (PAUF),CFPAC-1_shCtrl (shCtrl), CFPAC-1_shPAUF (shPAUF), BxPC-3_shCtrl (shCtrl), and BxPC-3 shPAUF (shPAUF) cell lines, and used for Western blot analysis with p-FAK, FAK, p-paxillin, or paxillin antibody. $\beta$-Actin was used as a loading control. (B) Cell lysates of Panc-1_Mock (Mock), Panc-1_PAUF (PAUF), CFPAC-1_shCtrl (shCtrl) and CFPAC-1_shPAUF (shPAUF) cell lines were used for Western blot analysis with p-Src or Src antibody. (C) Adhesion activity of Panc-1 Mock and Panc-1 PAUF cells were measured in the presence of PP2 $(10 \mu \mathrm{M})$ or Herbimycin $(10 \mu \mathrm{M})$. (D) Panc-1_Mock (Mock) and Panc-1_PAUF (PAUF) cells were incubated with anti-PAUF or control antibody. Cell lysates were prepared and used for western blot analysis with p-Src, Src, p-FAK, FAK, p-paxillin, or paxillin antibody. $\beta$-Actin was used as a loading control. Data represent mean values \pm SE. ${ }^{*}, P<0.05$.

tion, FAK phosphorylates scaffolding molecules, such as paxillin, through formation of a FAK-Src complex (Webb et al., 2004). FAK is fully activated after recruiting Src and forming FAK-Src complex (Bolos et al., 2010). Both phosphorylation and expression of paxillin were enhanced in PAUFoverexpressing cells. Conversely, knockdown of PAUF led to reduced levels of activated and total paxillin (Figure $2 \mathrm{~A}$ ). As shown in Figure $2 \mathrm{~B}$, activity and expression of Src were also positively correlated with PAUF expression. We next determined whether the activation of PAUF-mediated signaling is responsible for increased pancreatic cancer cell adhesion. As shown in Figure 2C, PAUF-induced adhesiveness of Panc-1 cells was significantly attenuated upon treatment with the Src inhibitors, PP2 and Herbimycin. Treatment of Panc-1_PAUF cells with anti-PAUF antibody, which resulted in reduced adhesiveness as shown in Figure $1 \mathrm{C}$, reduced expression and phosphorylation
A

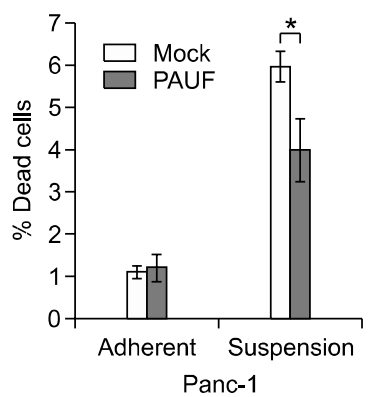

B

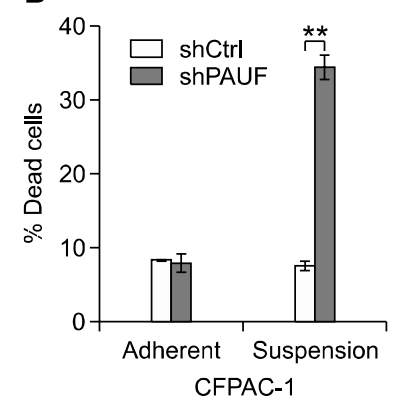

D
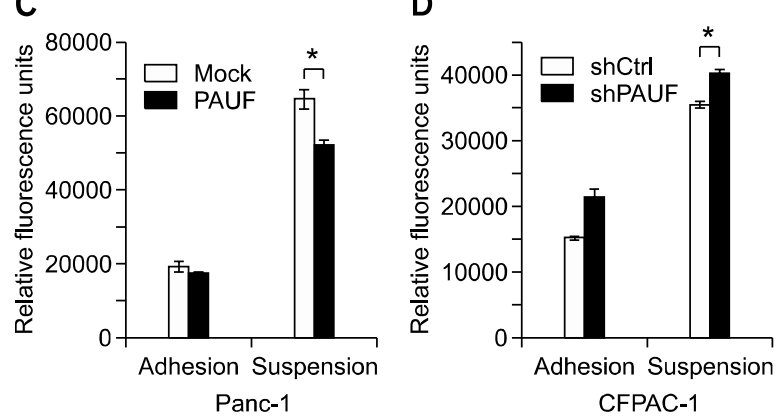

Figure 3. PAUF enhances resistance to anoikis. (A, B) Apoptosis of PAUF-regulated cells in adherent and suspension conditions was compared. PAUF-overexpressing (A) or knockdown (B) cells were seeded on poly-HEMA treated or untreated wells and the number of dead cells counted via trypan blue staining. (C, D) Caspase-3 activity in PAUF-overexpressing (C) or knockdown (D) cells was measured in poly-HEMA suspension conditions. The incubation time of these assay was $48 \mathrm{~h}$ in case of PAUF-overexpressing cells and $24 \mathrm{~h}$ in that of knockdown cells. Data represent mean values $\pm \mathrm{SE}$. ${ }^{*}, P<0.05,{ }^{* *}, P<$ 0.01 .

levels of Src, FAK, and paxillin (Figure 2D), supporting the involvement of FAK-Src signaling in this process.

\section{PAUF enhances resistance to anoikis}

Several studies have implicated FAK-Src signaling in resistance to anoikis, an apoptotic event triggered by inadequate or inappropriate cell-substrate contact (Zhao and Guan, 2009; Frame et al., 2010). Regulation of PAUF alters not only FAK-SrC signaling, but also cell-substrate contact. Accordingly, we examined whether PAUF affects anoikis induced in polyHEMA cultures of pancreatic cancer cells. As shown in Figures $3 \mathrm{~A}$ and $3 \mathrm{~B}$, resistance against anoikis was enhanced in PAUF-overexpressing cell lines (Panc-1_PAUF), but reduced in PAUF-downregulated cell lines (CFPAC-1_shPAUF). Activation of caspase 3 , mediated by anchorage deprivation, was significantly decreased upon overexpression of PAUF (Figure 3C). Consistently, suppression of PAUF enhanced anoikis-derived caspase 3 activity (Figure 3D). These results suggest that PAUF can 
enhance resistance against anoikis via disruption of the apoptotic caspase cascade.

\section{PAUF increases focal adhesions}

To elucidate whether PAUF modulates the density of focal adhesion, the site of cell-matrix adhesion, we stained cells with anti-paxillin. Focal adhesions are large macromolecular assemblies that mediate mechanical force transduction, adhesion to ECM and regulatory signals (Fraley et al., 2010). Paxillin is a docking protein containing many proteinbinding modules that recruit and localize signaling molecules to the focal adhesion site (Schaller, 2001). FAK localizes to and regulates focal adhesion site by binding paxillin (Schaller, 2001). In our experiments, PAUF-overexpressing cells formed more focal adhesions (Figure 4A). Focal adhesion sites were observed in both peripheral and central regions in Panc-1_PAUF cells, while the majority of focal adhesion sites were localized in the periphery of Panc-1_Mock cells. The number of focal adhesions in Panc-1_PAUF, calculated using the Image $\mathrm{J}$ program, was increased nearly 3 -fold, compared with that in the Panc-1_Mock cell line (Figure 4B). Consistent with this finding, PAUF-overexpressing cells contained more polymerized F-actin than control cells. The majority of actin bundles in Panc1_Mock cells were relatively short and restricted to the periphery in control cells (Figure 4A). These findings indicate that PAUF facilitates focal adhesion formation.

\section{Discussion}

FAK is associated with normal cell growth responses as well as survival and metastasis of tumor cells (Brunton and Frame, 2008). The majority of human cancers, especially highly invasive metastatic neoplasms, show elevated expression and activation of FAK (van Nimwegen and van de Water, 2007; Brunton and Frame, 2008). Earlier reports have suggested that FAK increases cell-matrix area and focal adhesion, and suppresses anoikis in the tumor microenvironment (Duxbury et al., 2004; Zhao and Guan, 2009; Bolos et al., 2010). These properties appear to mediate tumor metastasis. Here, we provide five lines of evidence to support PAUF-induced potentiation of metastasis through expression and activation of FAK. First, expression of PAUF was positively correlated with adhesiveness of pancreatic cancer cells to several ECM molecules in vitro. Second, anoikis was reduced upon PAUF overexpression, but induced by knockdown. Third, PAUF stimulated the FAK signaling
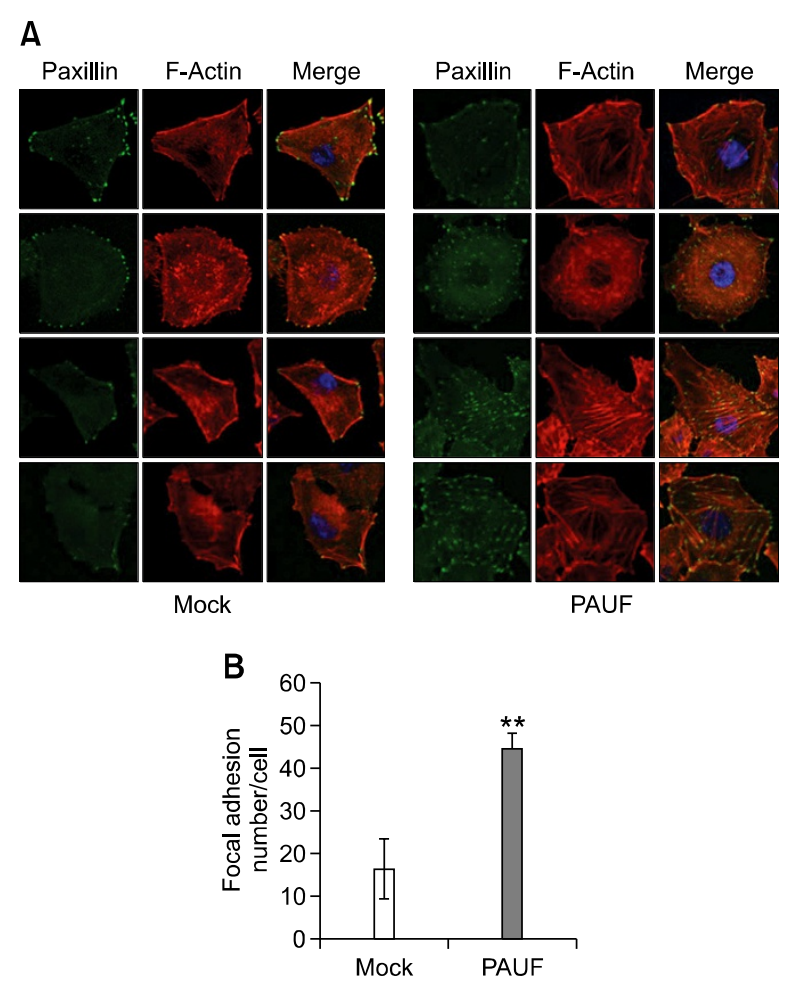

Figure 4. PAUF enhances the formation of focal adhesions. (A) Immunofluorescence staining of paxillin and F-actin was performed. Panc-1_Mock (Mock) and Panc-1_PAUF (PAUF) cells were fixed, permeabilized, and double-stained with anti-paxillin (green) and phalloidin-TRITC (red). (B) The number of focal adhesions stained with antipaxillin was calculated using the Image $\mathrm{J}$ program. Data represent mean values \pm SE. ${ }^{* *}, P<0.01$.

pathway at both the expression and activation levels. Fourth, both a specific antibody against PAUF and inhibitor of the FAK signaling pathway efficiently inhibited PAUF-mediated adhesiveness. Finally, the density of the focal adhesion site, represented by paxillin staining, was dramatically increased upon PAUF overexpression.

FAK is involved in multivalent aspects of tumorigenesis through transduction of intracellular signals associated with growth factor signaling and cellECM interactions (Sieg et al., 2000). In our experiments, overexpression of PAUF promoted both expression and activities of FAK and Src. For activation, FAK is autophosphorylated at its tyrosine residue 397 (Y397) where provided as a docking site for Src. The recruited Src phosphorylates additional sites on FAK and leads further activation. This activated FAK-Src complex regulates various signaling pathways and different cellular responses (Bolos et al., 2010). The MAPK pathway is one of the well-known downstream pathways of the FAK-Src complex. Phosphorylation of FAK by Src leads to recruitment of proteins containing $\mathrm{SH} 2$ 
domains, such as Grb2. Grb2 bound to FAK recruits SOS, and activates the Ras-MAPK pathway (Mitra and Schlaepfer, 2006; Bolos et al., 2010). Interactions of FAK and the p85 subunit of PI3K mediate activation of Akt kinase, which, in turn, protects cells from apoptosis (Reiske et al., 1999; Sonoda et al., 2000; Bolos et al., 2010). FAK additionally activates the JNK pathway, which promotes cell motility and invasiveness (Hsia et al., 2003; Liu et al., 2007). Previous analyses by our group have demonstrated that ERK, JNK and AKT are activated by overexpression of PAUF in pancreatic cancer cells (Lee et al., 2010). It is possible that FAK regulated by PAUF activates the ERK, JNK and AKT pathways. We are currently investigating the possibility in detail.

The activation mode of FAK can be classified into two groups. One is anchorage-dependent activation. Cell adhesion mediated by integrins facilitates anchorage-dependent cell growth through triggering tyrosine phosphorylation of key signaling molecules, including FAK. The other mode is anchorage-independent activation of FAK. When cells lose contact with the ECM, detachment-induced cell death, also known as anoikis, is triggered. However, in the case of transformed cells, abnormal FAK activation in the absence of adhesion signals contributes to anchorage-independent growth, a hallmark of cancerous cells (Zhao and Guan, 2009). Data from the present study showed that regulation of PAUF expression affects anoikis induced by polyHEMA culture. The increase in resistance against anoikis appears to originate from PAUFmediated FAK activation.

In conclusion, PAUF promotes cell adhesiveness to ECM molecules and protect cells from apoptosis induced by loss of anchorage. PAUF appears to contribute to metastasis of pancreatic cancer cells via induction of FAK expression and activation. Previous studies by our group have disclosed that PAUF facilitates the metastasis of pancreatic cancer cells via both autocrine and paracrine manner. The protein acts in an autocrine manner to enhance motility, invasiveness and proliferation of pancreatic cancer cells through induction of specific intracellular signaling cascades. PAUF mediates the transcriptional regulation of multiple tumor-related genes through activation of intracellular signaling. Analysis of pancreatic cancer cell lines and tissues of patients revealed close correlation of PAUF expression with that of CXCR4, a well-known metastatic factor (Li et al., 2004; Zlotnik, 2008; Lee et al., 2010). These findings indicate that secreted PAUF in pancreatic cancer cells enhances metastatic potential by upregulating CXCR4 expression in an autocrine manner. One recent study reported that CXCR4 is closely related with adhesiveness to ECM and anoikis of cancer cells (Kochetkova et al., 2009), suggesting that another mechanism of PAUF mediates attachment to ECM and survival. Secreted PAUF also acts on stromal cells in a paracrine manner. PAUF interacts with the CXCR4-TLR2 complex on the surface of monocytes, resulting in production of pro-tumorigenic cytokines and escape from innate immune surveillance (Park et al., 2011). Data from the current investigation have provided evidence that PAUF regulates interactions between pancreatic cancer cells and ECM molecules to facilitate cell adhesiveness and resistance against anoikis. Based on the collective findings, we propose that PAUF facilitates tumor growth and metastasis through regulation of not only tumor cells, but also the tumor microenvironment.

\section{Methods}

\section{Cell lines and materials}

The human pancreatic cancer cell lines Panc-1, MiaPaCa-2, CFPAC-1, and BxPC-3 were cultured in DMEM supplemented with $10 \%$ FBS, DMEM with $10 \%$ FBS and $2.5 \%$ horse serum, IMDM with $10 \%$ FBS, and RPMI with $10 \%$ FBS, respectively. All cell lines were obtained from the ATCC (Manassas, VA) and maintained in a humidified incubator at $37^{\circ} \mathrm{C}$ with $5 \% \mathrm{CO}_{2}$. Generation of PAUFoverexpressing (Panc-1_PAUF and MiaPaCa-2_PAUF) or -knockdown (CFPAC-1_shPAUF and BxPC-3_shPAUF) cell lines and their respective controls is described in a previous report (Lee et al., 2010). PP2 and herbimycin were from Calbiochem (San Diego, CA). Antibodies against Src, p-Src, FAK, p-FAK, paxillin and p-paxillin were from Cell Signaling. Anti- $\beta$-Actin antibody was from Santa Cruz (Santa Cruz, CA).

\section{Adhesion assay}

Cell adhesiveness was assayed using 96-well plate coated with fibronectin, collagen type I or vitronectin. Cells were seeded at a density of $1 \times 10^{4}$ cells/well in $100 \mu$ of serum-free medium, and then incubated at $37^{\circ} \mathrm{C}$. Unbound cells were removed by washing twice with PBS. Attached cells were fixed and stained with $0.5 \%$ crystal violet $/ 2 \%$ $\mathrm{EtOH} / 0.1 \mathrm{M}$ borated buffer ( $\mathrm{pH} 9.0$ ). Cell adhesion was quantified by counting the number of stained cells under a light microscope (Olympus). All assays were performed in triplicate and the tendencies confirmed with three independent experiments.

\section{Anoikis assay}

Anoikis was evaluated using nonadhesive polyHEMA (Sigma, Saint Louis, MO) coated 6 well plate. Cells $(1 \times$ $10^{5}$ cells/well) were seeded and incubated for $24 \mathrm{~h}$ (CFPAC-1) or $48 \mathrm{~h}$ (Panc- 1$)$ at $37^{\circ} \mathrm{C}$. Dead cells were 
detected using trypan blue staining.

\section{Caspase-3 activity assay}

Caspase-3 activity was determined using the specific substrates provided by the caspase assay kit, Ac-DEVDAMC (Peptron, Korea). Fluorophore (AMC) can be released and measured after these substrates have been cleaved by the caspase-3. After anoikis induction, cells were collected and resuspended in lysis buffer provided by the kit. After incubation with the corresponding substrate at $37^{\circ} \mathrm{C}$ for 30 min, fluorescence intensities were measured with the microplate fluorometer, FLUOstar OPTIMA (BMG Labtech, Germany).

\section{Western blot analysis}

Cells lysates were prepared in RIPA buffer and culture supernatants concentrated using Microcon centrifugal filters (Millipore, Billerica, MA). Samples were subjected to SDS-PAGE, and transferred to Hybond-ECL nitrocellulose membrane (Amersham, Buckinghamshire, Sweden). Membranes were probed with an appropriate primary antibody diluted in TBST containing 5\% BSA, followed by incubation with a secondary horseradish peroxidase-conjugated antibody. Bound antibody was visualized by using enhanced chemiluminescence reagents (Millipore, Billerica, MA).

\section{Evaluation of focal adhesion}

For immunofluorescence staining, $1 \times 10^{4}$ cells were seeded in Micro-Slide (Ibidi, Martinsried, Germany). Next, cells were fixed, followed by staining with anti-paxillin and phalloidin. After counterstaining with DAPI, cells were analyzed using LSM510 META confocal fluorescence microscope (Zeiss, Jena, Germany). The number of focal adhesions was counted using Image J.

\section{Statistical analysis}

Data were presented as mean values \pm standard errors (SE). Unless otherwise indicated, the student's $t$ test was used for statistical analysis, with $P<0.05$ defined as significant.

\section{Acknowledgements}

This work was supported by grants from the Inno-Project of the Korea Research Institute of Bioscience and Biotechnology and the $21^{\text {st }}$ Century Frontier Functional Human Genome Project of the Ministry of Education, Science and Technology, Korea.

\section{References}

Bolos V, Gasent JM, Lopez-Tarruella S, Grande E. The dual kinase complex FAK-Src as a promising therapeutic target in cancer. Onco Targets Ther 2010;3:83-97

Brunton VG, Frame MC. Src and focal adhesion kinase as therapeutic targets in cancer. Curr Opin Pharmacol 2008; 8:427-32

Cho IR, Koh SS, Min HJ, Kim SJ, Lee Y, Park EH, Ratakorn $\mathrm{S}$, Jhun $\mathrm{BH}$, Oh S, Johnston RN, Chung YH. Pancreatic adenocarcinoma up-regulated factor (PAUF) enhances the expression of $\beta$-catenin, leading to a rapid proliferation of pancreatic cells. Exp Mol Med 2011;43:82-90

Christofori G. New signals from the invasive front. Nature 2006;441:444-50

Duffy JP, Eibl G, Reber HA, Hines OJ. Influence of hypoxia and neoangiogenesis on the growth of pancreatic cancer. Mol Cancer 2003;2:12-21

Duxbury MS, Ito H, Zinner MJ, Ashley SW, Whang EE. Focal adhesion kinase gene silencing promotes anoikis and suppresses metastasis of human pancreatic adenocarcinom cells. Surgery 2004;135:555-62

Fraley SI, Feng Y, Krishnamurthy R, Kim DH, Celedon A, Longmore GD, Wirtz $\mathrm{D}$. A distinctive role for focal adhesion proteins in three dimensional cell motility. Nat Cell Biol 2010;12:598-604

Frame MC, Patel H, Serrels B, Lietha D, Eck MJ. The FERM domain: organizing the structure and function of FAK. Nat Rev Mol Cell Biol 2010;11:802-14

Garcea G, Dennison AR, Steward WP, Berry DP. Role of inflammation in pancreatic carcinogenesis and the implications for future therapy. Pancreatology 2005;5:514-29

Ghaneh P, Costello E, Neoptolemos JP. Biology and management of pancreatic cancer. Gut 2007;56:1134-52

Hsia DA, Mitra SK, Hauck CR, Streblow DN, Nelson JA, Ilic D, Huang S, Li E, Nemerow GR, Leng J, Spencer KS, Cheresh DA, Schlaepfer DD. Differential regulation of cell motility and invasion by FAK. J Cell Biol 2003;160:753-67

Kim SA, Lee Y, Jung DE, Park KH, Park JY, Gang J, Jeon SB, Park EC, Kim YG, Lee B, Liu Q, Zeng W, Yeramilli S, Lee S, Koh SS, Song SY. Pancreatic adenocarcinoma up-regulated factor (PAUF), a novel up-regulated secretory protein in pancreatic ductal adenocarcinoma. Cancer Sci 2009;100: 828-36

Kochetkova M, Kumar S, McColl SR. Chemokine receptors CXCR4 and CCR7 promote metastasis by preventing anoikis in cancer cells. Cell Death Differ 2009;16:664-73

Lark AL, Livasy CA, Calvo B, Caskey L, Moore DT, Yang X, Cance WG. Overexpression of focal adhesion kinase in primary colorectal carcinomas and colorectal liver metastases: immunohistochemistry and real-time PCR analyses. Clin Cancer Res 2003;9:215-22

Lee Y, Kim SJ, Park HD, Park EH, Huang SM, Jeon SB, Kim JM, Lim DS, Koh SS. PAUF functions in the metastasis of human pancreatic cancer cells and upregulates CXCR4 expression. Oncogene 2010;29:56-67

Li YM, Pan Y, Wei Y, Cheng X, Zhou BP, Tan M, Zhou X, Xia W, Hortobagyi GN, Yu D, Hung MC. Upregulation of CXCR4 is essential for HER2-mediated tumor metastasis. Cancer Cell 2004;6:459-69 
Liu S, Xu SW, Kennedy L, Pala D, Chen Y, Eastwood M, Carter DE, Black CM, Abraham DJ, Leask A. FAK is required for TGF $\beta$-induced JNK phosphorylation in fibroblasts: Implications for acquisition fo a matrix-remodeling phenotype. Mol Biol Cell 2007;18:2169-78

Maitra A, Hruban RH. Pancreatic cancer. Annu Rev Pathol 2008;3:157-88

Mitra SK, Schlaepfer DD. Integrin-regulated FAK-Src signaling in normal and cancer cells. Curr Opin Cell Biol 2006;18:516-23

Owens LV, Xu L, Craven RJ, Dent GA, Weiner TM, Kornberg L, Liu ET, Cance WG. Overexpression of the focal adhesion kinase (p125FAK) in invasive human tumors. Cancer Res 1995;55:2752-55

Park HD, Lee Y, Oh YK, Jung JG, Park YW, Myung K, Kim $\mathrm{KH}$, Koh SS, Lim DS. Pancreatic adenocarcinoma upregulated factor promotes metastasis by regulating TLR/CXCR4 activation. Oncogene 2011;30:201-11

Reiske HR, Kao SC, Cary LA, Guan JL, Lai JF, Chen HC. Requirement of phosphatidylinositol 3-kinase in focal adhesion kinase-promoted cell migration. J Biol Chem 1999;274:12361-66

Sahai E. Illuminating the metastatic process. Nat Rev Cancer 2007;7:737-49

Sawai H, Okada Y, Funahashi H, Matsuo Y, Takahashi H, Takeyama $\mathrm{H}$, Manabe T. Activation of focal adhesion kinase enhances the adhesion and invasion of pancreatic cancer cells via extracellular signal-regulated kinase-1/2 signaling pathway activation. Mol Cancer 2005;4:37

Schaller MD. Paxillin: a focal adhesion-associated adaptor protein. Oncogene 2001;20:6459-72

Sieg DJ, Hauck CR, llic D, Klingbeil CK, Schaefer E, Damsky $\mathrm{CH}$, Schlaepfer DD. FAK integrates growth-factor and integrin signals to promote cell migration. Nat Cell Biol 2000;2:249-56

Sonoda Y, Matsumoto Y, Funakoshi M, Yamamoto D, Hanks SK, Kasahara T. Anti-apoptotic role of FAK. Induction of inhibitor-of-apoptosis proteins and apoptosis suppression by the overexpression of FAK in a human leukemic cell line, HL-60. J Biol Chem 2000;275:16309-15

Steeg PS. Tumor metastasis: mechanistic insights and clinical challenges. Nat Med 2006;12:895-904

van Nimwegen MJ, van de Water B. Focal adhesion kinase: a potential target in cancer therapy. Biochem Pharmacol 2007;73:597-609

Webb DJ, Donais K, Whitmore LA, Thomas SM, Turner CE, Parsons JT, Horwitz AF. FAK-Src signaling through paxillin, ERK, and MLCK regulates adhesion disassembly. Nat Cell Biol 2004;6:154-61

Zhao J, Guan JL. Signal transduction by focal adhesion kinase in cancer. Cancer Metastasis Rev 2009;28:35-49

Zlotnik A. New insights on the role of CXCR4 in cancer metastasis. J Pathol 2008;215:211-13 